\title{
REGULARITY OF CURVES WITH A CONTINUOUS TANGENT LINE
}

\author{
J. M. BURGUÉS ${ }^{\bowtie}$ and J. CUFÍ \\ (Received 15 February 2007; accepted 27 July 2007) \\ Communicated by P. C. Fenton
}

\begin{abstract}
This note contains a proof of the fact that a Jordan curve in $\mathbb{R}^{2}$ with a continuous tangent line at each point admits a regular reparameterization. We extend the result both to more general curves in $\mathbb{R}^{n}$ and to higher orders of differentiability.
\end{abstract}

2000 Mathematics subject classification: primary 53A04; secondary 26E99, 30E99.

Keywords and phrases: regular curve, tangent line, Jordan curve, order of differentiability.

\section{Introduction}

An important result in the theory of the boundary regularity of the Riemann mapping, due to Lindelöf [Lin], asserts that a Jordan domain has a continuous tangent line at each point of the boundary if and only if the argument of the derivative of the Riemann mapping extends continuously to the boundary of the unit disk.

The traditional concept of a continuous tangent line at a point of a curve is of geometrical nature and essentially independent of the parameterization of the curve.

Definition 1. A Jordan curve $\gamma:[0,1] \rightarrow \mathbb{R}^{2}$ is said to have a continuous tangent line at each point if and only if there exists a continuous function $\beta:[0,1] \rightarrow \mathbb{R}$ satisfying, for any $t_{0}$,

$$
\lim _{t \rightarrow t_{0}^{+}} \arg \left\{\gamma(t)-\gamma\left(t_{0}\right)\right\}=\beta\left(t_{0}\right)
$$

and

$$
\lim _{t \rightarrow t_{0}^{-}} \arg \left\{\gamma(t)-\gamma\left(t_{0}\right)\right\}=\beta\left(t_{0}\right)+\pi
$$

The authors have been partially supported by MEC grant no. MTM2005-08984-CO and by grant no. 2005 SGR00611 of Generalitat de Catalunya.

(C) 2009 Australian Mathematical Society 1446-7887/2009 \$16.00 
(As usual, the argument is measured with respect to the $x$ axis in $\mathbb{R}^{2}$. Clearly the condition referred to is exact for $t_{0} \in(0,1)$ and has a different but analogous formulation for $t_{0}=0,1$.)

In the case of regular curves (having a $\mathcal{C}^{1}$ parameterization with nonvanishing tangent vector) the tangent line is given by the direction of the derivative.

The precise concept of regular curve comes from the following definitions.

Definition 2. A curve $\gamma:[0,1] \rightarrow \mathbb{R}^{n}$ is said to have a regular local parameterization if and only if:

(A) for any $t_{0} \in(0,1)$ there exist $\delta=\delta\left(t_{0}\right), J=J_{t_{0}} \subset \mathbb{R}$ a bounded open interval and $\mu: J \rightarrow \mathbb{R}^{n}, \mathcal{C}^{1}$, such that $\mu(J)=\gamma\left(t_{0}-\delta, t_{0}+\delta\right)$ and $\mu^{\prime}$ is never 0 on $J$;

(B) there exist $\delta_{0}^{\prime}>0, J_{0} \subset \mathbb{R}$ a bounded open interval and $\mu_{0}: J_{0} \rightarrow \mathbb{R}^{n}, \mathcal{C}^{1}$ such that $\mu_{0}\left(J_{0}\right)=\gamma\left(\left[0, \delta_{0}^{\prime}\right)\right) \cup \gamma\left(\left(1-\delta_{0}^{\prime}, 1\right]\right)$ and $\mu_{0}^{\prime}$ is never 0 on $J_{0}$.

\section{DEFINITION 3.}

(1) A curve $\gamma:[0,1] \rightarrow \mathbb{R}^{n}$ is said to have a regular global parameterization if and only if there exists $\mu:[0,1] \rightarrow \mathbb{R}^{n}$ realizing the properties (A) and (B).

(2) A curve $\gamma$ is said to be regular if and only if it has a global regular parameterization.

The assumption that the derivative is always nonzero is more subtle and basic than it appears on first sight. Every polygonal line permits an infinitely differentiable parameterization $\gamma$. The point is that $\gamma^{\prime}(t)=0$ for $t$ corresponding to a corner.

It is often taken for granted that the definitions of having a continuous tangent line and being regular are equivalent. For instance, in [Pom, Section 3.2] the geometric tangent definition is used to prove Lindelöf's theorem, whereas in [Pom, Section 3.3] the other definition is used.

The proof of the fact that regular curves possess a continuous tangent line is quite elementary. In the present paper we give an accessible proof of the converse. There cannot be any doubt that the classical literature contains a proof, but the authors were unable to find a reference.

However, our proof covers the case of general (not necessarily Jordan) curves in $\mathbb{R}^{n}$, as well as a generalization to higher orders of differentiability.

\section{Curves with continuous geometric tangent lines}

Suppose, now, that $\gamma:(0,1) \rightarrow \mathbb{R}^{n}$ is a continuous arc with the natural assumption that no open interval in $(0,1)$ is applied by $\gamma$ to a single point. In the rest of this paper, the notation for the components of a curve $\gamma$ will be $\gamma(t)=\left(\gamma_{1}(t), \ldots, \gamma_{n}(t)\right)$, as well as $\left(\right.$, ) for the standard scalar product in $\mathbb{R}^{n}$.

Definition 4 (Continuous geometric tangent line). We will say that $\gamma$ has a continuous tangent line at each point if and only if there is a continuous 
map $B:(0,1) \rightarrow S^{n-1}$ (the Euclidean unit sphere in $\mathbb{R}^{n}$ ) such that, for any $t_{0} \in(0,1)$,

$$
\lim _{t \rightarrow t_{0}^{+}} \frac{\gamma(t)-\gamma\left(t_{0}\right)}{\left\|\gamma(t)-\gamma\left(t_{0}\right)\right\|}=B\left(t_{0}\right)
$$

and

$$
\lim _{t \rightarrow t_{0}^{-}} \frac{\gamma(t)-\gamma\left(t_{0}\right)}{\left\|\gamma(t)-\gamma\left(t_{0}\right)\right\|}=-B\left(t_{0}\right)
$$

whenever $\gamma(t) \neq \gamma\left(t_{0}\right)$.

REMARK 5. Observe that under the hypotheses of the definition above, any point in the curve has finite multiplicity. Otherwise there would be a point $t_{0} \in(0,1)$ and a sequence of disjoint open intervals $I_{l}=\left(\alpha_{l}, \beta_{l}\right)$ whose extreme points increase (or decrease) to $t_{0}$ and satisfy $\gamma\left(\alpha_{l}\right)=\gamma\left(\beta_{l}\right)=\gamma\left(t_{0}\right)$, for any $l$. It is possible to find a sequence of points $s_{l} \in I_{l}$ such that

$$
\left(\left(\gamma\left(s_{l}\right)-\gamma\left(\alpha_{l}\right)\right) /\left(\left\|\gamma\left(s_{l}\right)-\gamma\left(\alpha_{l}\right)\right\|\right)\right)=B\left(\alpha_{l}\right)+w_{l} \quad \text { and } \quad\left\|w_{l}\right\| \rightarrow_{l \rightarrow+\infty} 0 .
$$

This means that

$$
\left(\left(\left(\gamma\left(s_{l}\right)-\gamma\left(\alpha_{l}\right)\right) /\left(\left\|\gamma\left(s_{l}\right)-\gamma\left(\alpha_{l}\right)\right\|\right)\right), \quad B\left(t_{0}\right)\right) \rightarrow_{t \rightarrow+\infty} 1,
$$

but

$$
\begin{aligned}
& \left(\left(\gamma\left(s_{l}\right)-\gamma\left(\alpha_{l}\right)\right) /\left(\left\|\gamma\left(s_{l}\right)-\gamma\left(\alpha_{l}\right)\right\|\right)\right) \\
& \quad=\left(\left(\gamma\left(s_{l}\right)-\gamma\left(t_{0}\right)\right) /\left(\left\|\gamma\left(s_{l}\right)-\gamma\left(t_{0}\right)\right\|\right)\right) \rightarrow-B\left(t_{0}\right),
\end{aligned}
$$

so the limit of the scalar product above should be -1 . This is a contradiction.

The previous definition is the one adopted in [Gar-Mar, p. 60] for Jordan curves, in the case of $n=2$.

Even in $\mathbb{R}^{n}$, the definition above imposes strong restrictions on the curve.

PROPOSITION 6. If $\gamma$ has a continuous tangent line at each point, then for every $t_{0} \in(0,1)$ there exist $\delta>0$ and $j \in\{1, \ldots, n\}$ such that $\gamma_{j}:\left(t_{0}-\delta, t_{0}+\delta\right) \rightarrow \mathbb{R}$ is injective.

PROOF. For a fixed $t_{0} \in(0,1)$, after an affine change of coordinates, we may assume that $B\left(t_{0}\right)=(1,0, \ldots, 0)=e_{1}$. Then there exists $\delta>0$ such that $\left(B(t), e_{1}\right)>0$ for $t \in\left(t_{0}-\delta, t_{0}+\delta\right)$. As a consequence $\gamma_{1}(t)$ is injective on this interval, otherwise there would be $a, b \in\left(t_{0}-\delta, t_{0}+\delta\right)$ such that $\gamma_{1}(a)=\gamma_{1}(b)$, and this would imply the existence of a point $\tau \in(a, b)$ with $\gamma_{1}(\tau)=\gamma_{1}(a)=\gamma_{1}(b)$.

For $t$ in a neighborhood of $a$ and $t>a,\left(\gamma(t)-\gamma(a), e_{1}\right)>0$, which implies that $\gamma_{1}(t)>\gamma_{1}(a)=\gamma_{1}(b)$. Analogously, for $t$ in a neighborhood of $b$ and $t<b$, we have $\left(\gamma(t)-\gamma(b), e_{1}\right)<-\frac{1}{2}\left(B(b), e_{1}\right)<0$, and therefore $\gamma_{1}(t)<\gamma_{1}(b)=\gamma_{1}(a)$. Then Bolzano's theorem applied to the function $f(t)=\gamma_{1}(t)-\gamma_{1}(a)$ will show the existence of $\tau$.

Iteration of this procedure provides points $\tau_{n} \rightarrow \tau_{0}$ with $\tau_{n}, \tau_{0} \in\left(t_{0}-\delta, t_{0}+\delta\right)$ such that $\gamma_{1}\left(\tau_{n}\right)=\gamma_{1}\left(\tau_{0}\right)$. Then $\left(\gamma\left(\tau_{n}\right)-\gamma\left(\tau_{0}\right), e_{1}\right)=0$, but $\left(B\left(\tau_{0}\right), e_{1}\right)>0$. 
COROLlary 7. If $\gamma:[0,1] \rightarrow \mathbb{R}^{n}$ is a curve having a continuous tangent line at every point, then for any $t_{0} \in[0,1]$ there is an open neighborhood $I_{t_{0}}$ (in the extended sense for the cases $\left.t_{0}=0,1\right)$, such that $\gamma_{\mid I_{t_{0}}}$ is a Jordan arc.

\section{The case of Jordan arcs}

Suppose now that $\gamma:[0,1] \rightarrow \mathbb{R}^{n}$ is a Jordan arc ( $\gamma$ continuous and injective).

PROPOSITION 8. If $\gamma$ has a continuous tangent line at each point, then the set $\gamma((0,1))$ admits a regular local parameterization.

PROOF. We proceed by induction on the dimension.

Fix $t_{0} \in(0,1)$. After a rigid movement in $\mathbb{R}^{n}$ we may suppose that $\gamma\left(t_{0}\right)=0$ and $B\left(t_{0}\right)=e_{1}$. By Proposition 6 , there exists $\delta>0$ such that $\gamma_{1}:\left(t_{0}-\delta, t_{0}+\delta\right) \rightarrow \mathbb{R}$ is an injective map to the $X_{1}$ axis, and so it is the projection of $\gamma\left(\left(t_{0}-\delta, t_{0}+\delta\right)\right)$ onto the hyperplane $\left\langle e_{n}\right\rangle_{\mathbb{R}}^{\perp}$. Let $p: \mathbb{R}^{n} \rightarrow\left\langle e_{n}\right\rangle_{\mathbb{R}}^{\perp}$ be the orthogonal projection map.

Since $p$ is continuous, $p \circ \gamma$ is a continuous curve, and since $t \rightarrow\left(p \circ \gamma(t), e_{1}\right)=$ $\gamma_{1}(t)$ is injective in $I=\left(t_{0}-\delta, t_{0}+\delta\right)$, so is $\rho=p \circ \gamma$ in this interval.

Then $\rho$ is a Jordan arc in $\mathbb{R}^{n-1}$. Moreover, if $t_{1} \in I$, then

$$
\begin{aligned}
p\left(B\left(t_{1}\right)\right) & =p\left(\lim _{t \rightarrow t_{1}^{+}} \frac{\gamma(t)-\gamma\left(t_{1}\right)}{\left\|\gamma(t)-\gamma\left(t_{1}\right)\right\|}\right)=\lim _{t \rightarrow t_{1}^{+}} \frac{p \circ \gamma(t)-p \circ \gamma\left(t_{1}\right)}{\left\|\gamma(t)-\gamma\left(t_{1}\right)\right\|} \\
& =\lim _{t \rightarrow t_{1}^{+}} \frac{\rho(t)-\rho\left(t_{1}\right)}{\left\|\gamma(t)-\gamma\left(t_{1}\right)\right\|},
\end{aligned}
$$

and therefore

$$
\left\|p\left(B\left(t_{1}\right)\right)\right\|=\lim _{t \rightarrow t_{1}^{+}} \frac{\left\|\rho(t)-\rho\left(t_{1}\right)\right\|}{\left\|\gamma(t)-\gamma\left(t_{1}\right)\right\|} .
$$

Since $\left(B\left(t_{1}\right), e_{1}\right) \neq 0$, we see that $\left\|p\left(B\left(t_{1}\right)\right)\right\|>0$, and

$$
\begin{aligned}
\lim _{t \rightarrow t_{1}^{+}} \frac{\rho(t)-\rho\left(t_{1}\right)}{\left\|\rho(t)-\rho\left(t_{1}\right)\right\|} & =\lim _{t \rightarrow t_{1}^{+}} \frac{p \circ \gamma(t)-p \circ \gamma\left(t_{1}\right)}{\left\|\gamma(t)-\gamma\left(t_{1}\right)\right\|} \lim _{t \rightarrow t_{1}^{+}} \frac{\left\|\gamma(t)-\gamma\left(t_{1}\right)\right\|}{\left\|\rho(t)-\rho\left(t_{1}\right)\right\|} \\
& =\lim _{t \rightarrow t_{1}^{+}} \frac{\left\|\gamma(t)-\gamma\left(t_{1}\right)\right\|}{\left\|\rho(t)-\rho\left(t_{1}\right)\right\|} \lim _{t \rightarrow t_{1}^{+}} p\left(\frac{\gamma(t)-\gamma\left(t_{1}\right)}{\left\|\gamma(t)-\gamma\left(t_{1}\right)\right\|}\right)
\end{aligned}
$$

because $p$ is linear, so

$$
\lim _{t \rightarrow t_{1}^{+}} \frac{\rho(t)-\rho\left(t_{1}\right)}{\left\|\rho(t)-\rho\left(t_{1}\right)\right\|}=p\left(B\left(t_{1}\right)\right) \lim _{t \rightarrow t_{1}^{+}} \frac{\left\|\gamma(t)-\gamma\left(t_{1}\right)\right\|}{\left\|\rho(t)-\rho\left(t_{1}\right)\right\|}=p\left(B\left(t_{1}\right)\right) \frac{1}{\left\|p\left(B\left(t_{1}\right)\right)\right\|} \neq 0 .
$$

Now, if the result is true for Jordan arcs in $\mathbb{R}^{n-1}$, then $\rho$ admits a local $\mathcal{C}^{1}$ parameterization. Let us call it

$$
\mu:\left(\tau_{0}-\delta^{\prime \prime}, \tau_{0}+\delta^{\prime \prime}\right) \rightarrow \mathbb{R}^{n-1},
$$

with $\mu\left(\tau_{0}\right)=\rho\left(t_{0}\right)$, for some $\delta^{\prime \prime}>0$. 
On the other hand, the injectivity of the projection $p$ in $\rho(I)$ implies that for a small interval $I_{0} \Subset I, \gamma\left(I_{0}\right)$ is a graph over $\rho\left(I_{0}\right)$, that is, there exists a function $f: \rho\left(I_{0}\right) \rightarrow \mathbb{R}$ such that

$$
\gamma\left(I_{0}\right)=\left\{(\mu(\tau), f(\mu(\tau))): \tau \in\left(\tau_{0}-\delta^{\prime \prime}, \tau_{0}+\delta^{\prime \prime}\right)\right\} .
$$

The parameterization $\tau \rightarrow(\mu(\tau), f(\mu(\tau)))$ is $\mathcal{C}^{1}$ in $\left(\tau_{0}-\delta^{\prime \prime}, \tau_{0}+\delta^{\prime \prime}\right)$ because, for any $t_{1} \in\left(\tau_{0}-\delta^{\prime \prime}, \tau_{0}+\delta^{\prime \prime}\right)$,

$$
\frac{f\left(\mu\left(\tau_{1}+h\right)\right)-f\left(\mu\left(\tau_{1}\right)\right)}{h}=\frac{f\left(\mu\left(\tau_{1}+h\right)\right)-f\left(\mu\left(\tau_{1}\right)\right)}{\left\|\mu\left(\tau_{1}+h\right)-\mu\left(\tau_{1}\right)\right\|} \frac{\left\|\mu\left(\tau_{1}+h\right)-\mu\left(\tau_{1}\right)\right\|}{h} .
$$

The first term is

$$
\begin{aligned}
& \frac{\gamma_{n}\left(t_{1}+s\right)-\gamma_{n}\left(t_{1}\right)}{\left\|p\left(\gamma\left(t_{1}+s\right)\right)-p\left(\gamma\left(t_{1}\right)\right)\right\|} \\
& \quad=\frac{\left(\left(\gamma_{n}\left(t_{1}+s\right)-\gamma_{n}\left(t_{1}\right)\right) /\left(\left\|\gamma\left(t_{1}+s\right)-\gamma\left(t_{1}\right)\right\|\right)\right)}{\left(\left(\left\|p\left(\gamma\left(t_{1}+s\right)\right)-p\left(\gamma\left(t_{1}\right)\right)\right\|\right) /\left(\left\|\gamma\left(t_{1}+s\right)-\gamma\left(t_{1}\right)\right\|\right)\right)},
\end{aligned}
$$

having limit

$$
\frac{B_{n}\left(t_{1}\right)}{\left\|p\left(B\left(t_{1}\right)\right)\right\|}
$$

as $h \rightarrow 0^{+}$(or $s \rightarrow 0^{+}$). The second term has limit $\left\|\mu^{\prime}\left(\tau_{1}\right)\right\|$. Then

$$
\lim _{h \rightarrow 0^{+}} \frac{f\left(\mu\left(\tau_{1}+h\right)\right)-f\left(\mu\left(\tau_{1}\right)\right)}{h}=\frac{B_{n}\left(\gamma^{-1}\left(\tau_{1}\right)\right)}{\| p\left(B\left(\gamma^{-1}\left(\tau_{1}\right)\right) \|\right.}\left\|\mu^{\prime}\left(\tau_{1}\right)\right\| .
$$

The limit when $h \rightarrow 0^{-}$can be managed in a similar way.

Also, this parameterization has nonvanishing tangent vector, because $\mu^{\prime}(\tau)$ is never 0 .

The case $n=1$ is trivial, and this, as the first induction step, would conclude the assertion. Nevertheless, we begin the induction with the case of $n=2$ because it contains the basic ingredients of the general proof, and is also in itself of interest as a standard statement in the study of the boundary regularity of the Riemann conformal map. In this case, the usual presentation of the hypotheses uses the function $\beta(t)=\arctan \left(\left(B_{2}(t)\right) /\left(B_{1}(t)\right)\right)$. We will make temporary use of this notation.

Since $\gamma$ is continuous, it follows that $J=\gamma_{1}\left(t_{0}-\delta, t_{0}+\delta\right)$ is an open interval of the $X$ axis, and the set $\left\{\gamma(t): t \in\left(t_{0}-\delta, t_{0}+\delta\right)\right\}$ is the graph of the function $f(x)=\gamma_{2} \circ \gamma_{1}^{-1}(x)$, defined in $J$.

Now, $f$ is a $\mathcal{C}^{1}$ function on $J$ : if $x_{0} \in J, x_{0}=\gamma_{1}\left(t_{1}\right)$, then

$$
\lim _{h \rightarrow 0}\left\{\frac{f\left(x_{0}+h\right)-f\left(x_{0}\right)}{h}-\tan \beta\left(t_{0}\right)\right\}=\lim _{t \rightarrow t_{0}}\left\{\frac{\gamma_{2}(t)-\gamma_{2}\left(t_{1}\right)}{\gamma_{1}(t)-\gamma_{1}\left(t_{1}\right)}-\tan \beta\left(t_{1}\right)\right\}=0,
$$

so, since $\beta$ is a continuous function, then $f \in \mathcal{C}^{1}$, and $x \rightarrow(x, f(x))$ is a $\mathcal{C}^{1}$ parameterization of the curve $\gamma$ around the point $\gamma\left(t_{0}\right)$, with nonvanishing tangent vector $\left(1, f^{\prime}(x)\right)$. (In fact $\left.f^{\prime}(x)=\tan \beta\left(\gamma_{1}^{-1}(x)\right)\right)$.) 


\section{Globalization of the parameterization}

Curves possessing a local $\mathcal{C}^{1}$ parameterization also have a global one in a natural way.

PROPOSITION 9. If $\gamma$ is a continuous closed curve in $\mathbb{R}^{n}$ having a regular local parameterization, then $\gamma$ admits a regular global parameterization.

PRoof. Let $t_{0} \in(0,1)$ and $\varsigma_{0}=\gamma\left(t_{0}\right)$. By the Proposition 8 , there are intervals $I_{t_{0}} \Subset$ $(0,1), J \Subset \mathbb{R}$ and $\mu: J \rightarrow \mathbb{R}^{n}$ a local $\mathcal{C}^{1}$ parameterization of $\gamma(I)$ with nonvanishing derivative. We can choose $\tau_{0} \in J$ such that $\mu\left(\tau_{0}\right)=\varsigma_{0}$, and since $\mu^{\prime}\left(\tau_{0}\right) \neq 0$, there is an open interval $J_{\tau_{0}}^{\prime} \Subset J$ where $\mu$ is injective, and $\mu\left(J^{\prime}\right)$ coincides with the image by $\gamma$ of a corresponding interval $I^{\prime}$, as in cases (A) and (B) of the definition in Section 1.

(Without loss of generality, we may suppose that the first component of $\mu^{\prime}\left(\tau_{0}\right)$ is strictly positive, and so the first component of $\mu$ is an homeomorphism from an open interval $J_{\tau_{0}}^{\prime}$ to an open interval $K \subset \mathbb{R}$ containing the image of $\tau_{0}$ in its interior. Then $\gamma^{-1}(K)$ contains $t_{0}$ in its interior, and we choose the corresponding interval.)

A similar procedure works for $t_{0}=0$ or 1 .

To show that the curve $\gamma$ is rectifiable, take a finite covering of $[0,1]$ by intervals such that the image admits a parameterization $\mu$ in a neighborhood of the closure of $J$ ( $\mu$ and $J$ as above). Each $\operatorname{arc} \mu(J)$ has finite length, therefore so does $\gamma([0,1])$. Let $L>0$ be the length of $\gamma([0,1])$.

Moreover, there is a finite collection of points $0<t_{1}<\cdots<t_{p}<1$ and positive numbers $\delta_{1}, \ldots, \delta_{p}, \delta^{\prime}$ for which there is an associated covering of $[0,1]$ by intervals

$$
I_{0}=\left[0, \delta^{\prime}\right), \ldots, I_{j}=\left(t_{j}-\delta_{j}, t_{j}+\delta_{j}\right), \ldots, I_{p+1}=\left(1-\delta^{\prime}, 1\right],
$$

such that $I_{j}$ only intersects $I_{j-1}, I_{j+1}$.

Choose points $t_{j}^{\prime} \in I_{j} \cap I_{j+1}$, for $j=0, \ldots, p$, and consider the arcs $\Gamma_{0}=$ $\gamma\left(\left[0, t_{0}^{\prime}\right] \cup\left[t_{p}^{\prime}, 1\right]\right)$ and $\Gamma_{j}=\gamma\left(\left[t_{j}^{\prime}, t_{j+1}^{\prime}\right]\right)$. Also let $x_{j}=\gamma\left(t_{j}^{\prime}\right)$.

For any $j$, for the corresponding $J_{j}$ and $\mu_{j}, \Gamma_{j} \subset \mu_{j}\left(J_{j}\right)$, and since $\mu_{j}$ is continuous and injective in $J_{j}$, we can parameterize $\Gamma_{j}$ by its arc length:

$$
s_{j}(\tau)=\int_{\mu_{j}^{-1}\left(x_{j}\right)}^{\tau}\left\|\mu_{j}^{\prime}(\xi)\right\| d \xi
$$

and $\lambda_{j}\left(s_{j}\right)=\mu_{j}\left(\tau\left(s_{j}\right)\right)$, for $s_{j} \in\left[0, \ell\left(\Gamma_{j}\right)\right)$, where $\ell$ denotes length. Then we have a global parameterization.

In $[0, L]$ we consider the points $\sigma_{j}=\sum_{k=1}^{j} \ell\left(\Gamma_{k}\right)$. On each interval $\left[L_{j}, L_{j+1}\right]$, we consider the parameterization $\lambda_{j}$, and define $\varrho:[0, L] \rightarrow \mathbb{R}^{n}$ as $\varrho(s)=\lambda_{j}\left(s-L_{j}\right)$, for $s \in\left[L_{j}, L_{j+1}\right]$. The fact that $\left\|\varrho^{\prime}\right\| \equiv 1$ and that the direction of $\varrho^{\prime}$ is the same as the corresponding $\mu_{j}^{\prime}$, which are continuous, imply that $\varrho$ is globally $\mathcal{C}^{1}$.

We conclude this section with the following corollary.

THEOREM 10. If $\gamma:[0,1] \rightarrow \mathbb{R}^{n}$ is a (continuous closed) curve having a continuous tangent line at each point, then $\gamma$ admits a regular global parameterization. 
REMARK 11. The case $n=2$ provides the classical statement that the Jordan curves in $\mathbb{R}^{2}$ having a continuous tangent line at each point are regular.

\section{Higher order of differentiability}

The previous sections have demonstrated that the geometrical condition of having a tangent line at each point implies that the curve admits a $\mathcal{C}^{1}$ parameterization with nonvanishing derivative, and that this geometrical property is independent of the particular parameterization $\gamma$, that is, it can be checked from an a priori given parameterization $\gamma$. We now study how the existence of reparameterizations of higher order of differentiability can be also checked by looking at the original parameterization.

We begin with the fact that a curve satisfying the condition

$$
\lim _{t \rightarrow t_{0}^{ \pm}}\left(\left(\gamma(t)-\gamma\left(t_{0}\right)\right) /\left(\left\|\gamma(t)-\gamma\left(t_{0}\right)\right\|\right)\right)= \pm B\left(t_{0}\right),
$$

with $B(t)$ continuous, admits a parameterization $\rho$ by the $\operatorname{arc}$ length, which is $\mathcal{C}^{1}$. Then it is an easy observation that the curve admits a $\mathcal{C}^{k}$ parameterization if and only if $\rho$ is $\mathcal{C}^{k}$.

Let us consider the case $k=2$. The curve admits a $\mathcal{C}^{2}$ parameterization if and only if the limit

$$
\lim _{s \rightarrow s_{0}} \frac{\varrho^{\prime}(s)-\varrho^{\prime}\left(s_{0}\right)}{s-s_{0}}=\varrho^{\prime \prime}\left(s_{0}\right)
$$

is continuous.

Fix $t_{0}$ and $t$. Since there exists a $\mathcal{C}^{1}$ diffeomorphism $\theta$ such that $s=\theta(t)$, then

$$
\begin{aligned}
& \frac{\varrho^{\prime}(s)-\varrho^{\prime}\left(s_{0}\right)}{s-s_{0}} \\
& \quad=\frac{\varrho^{\prime}(\theta(t))-\varrho^{\prime}\left(\theta\left(t_{0}\right)\right)}{\theta(t)-\theta\left(t_{0}\right)}=\frac{B(t)-B\left(t_{0}\right)}{\theta(t)-\theta\left(t_{0}\right)} \\
& \quad=\frac{1}{\theta(t)-\theta\left(t_{0}\right)}\left\{\sigma\left(\tau^{\prime}, t\right) \frac{\gamma\left(\tau^{\prime}\right)-\gamma(t)}{\left\|\gamma\left(\tau^{\prime}\right)-\gamma(t)\right\|}+\sigma\left(\tau, t_{0}\right) \frac{\gamma(\tau)-\gamma\left(t_{0}\right)}{\left\|\gamma(\tau)-\gamma\left(t_{0}\right)\right\|}+w\left(\tau^{\prime}, \tau, t, t_{0}\right)\right\},
\end{aligned}
$$

where $w=o\left(\left|\theta(t)-\theta\left(t_{0}\right)\right|\right)$ and $\sigma\left(t^{\prime}, t^{\prime \prime}\right)=1$ if $t^{\prime \prime}<t^{\prime}$ and -1 if $t^{\prime}<t^{\prime \prime}$.

Since the curve is rectifiable,

$$
\theta(t)-\theta\left(t_{0}\right)=s-s_{0}=\sup \left\{\sum_{i}\left\|\gamma\left(\tau_{i}\right)-\gamma\left(\tau_{i-1}\right)\right\| ;\left\{\tau_{i}\right\} \subset \mathcal{P}\left(J_{t, t_{0}}\right)\right\},
$$

where $\mathcal{P}\left(J_{t, t_{0}}\right)$ is the set of partitions of the interval between $t$ and $t_{0}$. Then, for a given $0<\epsilon<\left(s-s_{0}\right)^{2}$, there is a partition $\left\{\tau_{i}\right\}$ such that

$$
\left\|\gamma(t)-\gamma\left(t_{0}\right)\right\| \leq s-s_{0}=\alpha+\sum_{i}\left\|\gamma\left(\tau_{i}\right)-\gamma\left(\tau_{i-1}\right)\right\|,
$$

where $|\alpha|<\epsilon$. 
The main condition on the curve implies that

$$
\gamma\left(\tau_{i}\right)-\gamma\left(\tau_{i-1}\right)=\left(B\left(t_{0}\right)+v_{i}\right)\left\|\gamma\left(\tau_{i}\right)-\gamma\left(\tau_{i-1}\right)\right\|,
$$

where $\left\|v_{i}\right\|=o(1)$, for $\left|t-t_{0}\right|$ small. So

$$
\begin{aligned}
\gamma(t)-\gamma\left(t_{0}\right) & =\sum_{i} \gamma\left(\tau_{i}\right)-\gamma\left(\tau_{i-1}\right) \\
& =B\left(t_{0}\right) \sum_{i}\left\|\gamma\left(\tau_{i}\right)-\gamma\left(\tau_{i-1}\right)\right\|+\sum_{i}\left\|\gamma\left(\tau_{i}\right)-\gamma\left(\tau_{i-1}\right)\right\| v_{i} \\
& =\left(s-s_{0}\right) B\left(t_{0}\right)-\alpha B\left(t_{0}\right)+\sum_{i}\left\|\gamma\left(\tau_{i}\right)-\gamma\left(\tau_{i-1}\right)\right\| v_{i}
\end{aligned}
$$

and

$$
\left(\gamma(t)-\gamma\left(t_{0}\right), B\left(t_{0}\right)\right)=\left(s-s_{0}\right)(1+o(1)) .
$$

This implies that

$$
\left(\frac{\gamma(t)-\gamma\left(t_{0}\right)}{\left\|\gamma(t)-\gamma\left(t_{0}\right)\right\|}, B\left(t_{0}\right)\right)=\frac{s-s_{0}}{\left\|\gamma(t)-\gamma\left(t_{0}\right)\right\|}(1+o(1)),
$$

and so

$$
\lim _{t \rightarrow t_{0}} \frac{s-s_{0}}{\left\|\gamma(t)-\gamma\left(t_{0}\right)\right\|}=1 .
$$

Then

$$
\begin{aligned}
\lim _{s \rightarrow s_{0}} \frac{\varrho^{\prime}(s)-\varrho^{\prime}\left(s_{0}\right)}{s-s_{0}}= & \lim _{t \rightarrow t_{0}} \frac{B(t)-B\left(t_{0}\right)}{\left\|\gamma(t)-\gamma\left(t_{0}\right)\right\|}=\lim _{t \rightarrow t_{0}} \frac{\sigma\left(t, t_{0}\right)}{\left\|\gamma(t)-\gamma\left(t_{0}\right)\right\|} \\
& \times\left\{\sigma\left(\tau^{\prime}, t\right) \frac{\gamma\left(\tau^{\prime}\right)-\gamma(t)}{\left\|\gamma\left(\tau^{\prime}\right)-\gamma(t)\right\|}+\sigma\left(\tau, t_{0}\right) \frac{\gamma(\tau)-\gamma\left(t_{0}\right)}{\left\|\gamma(\tau)-\gamma\left(t_{0}\right)\right\|}\right\},
\end{aligned}
$$

which gives the result.

THEOREM 12. If $\gamma:[0,1] \rightarrow \mathbb{R}^{n}$ is a continuous closed curve, then $\gamma$ admits a $\mathcal{C}^{2}$ parameterization with nonvanishing first derivative if and only if there are two vector-valued continuous functions

$$
B^{(j)}:[0,1] \rightarrow \mathbb{R}^{n}, \quad j=1,2,
$$

such that

$$
B^{(1)}\left(t_{0}\right)=\lim _{t \rightarrow t_{0}} \frac{1}{\left\|\gamma(t)-\gamma\left(t_{0}\right)\right\|}\left\{\sigma\left(t, t_{0}\right)\left(\gamma(t)-\gamma\left(t_{0}\right)\right)\right\} \neq 0
$$

and

$$
\begin{aligned}
B^{(2)}\left(t_{0}\right)= & \lim _{t \rightarrow t_{0} ;\left|\tau^{\prime}-t\right|,\left|\tau-t_{0}\right|=o\left(\left|t-t_{0}\right|\right)} \frac{\sigma\left(t, t_{0}\right)}{\left\|\gamma(t)-\gamma\left(t_{0}\right)\right\|}\left\{\sigma\left(\tau^{\prime}, t\right) \frac{\gamma\left(\tau^{\prime}\right)-\gamma(t)}{\left\|\gamma\left(\tau^{\prime}\right)-\gamma(t)\right\|}\right. \\
& \left.+\sigma\left(\tau, t_{0}\right) \frac{\gamma(\tau)-\gamma\left(t_{0}\right)}{\left\|\gamma(\tau)-\gamma\left(t_{0}\right)\right\|}\right\},
\end{aligned}
$$

where the precedence indicator $\sigma(\alpha, \beta)$ is 1 if $\beta<\alpha$ and -1 if $\alpha<\beta$. 
REMARK 13. In this case, if $\gamma$ is parameterized by the arc length, the term $B^{(2)}$ corresponds to the curvature parameters, so is the curvature radius and the normal vector.

Then, if these parameters are given a priori and are continuous in the tangent direction, then $\gamma$ admits a $\mathcal{C}^{2}$ parameterization.

The corresponding statement for the $\mathcal{C}^{k}$ case is as follows.

THEOREM 14. If $\gamma:[0,1] \rightarrow \mathbb{R}^{n}$ is a continuous closed curve, then $\gamma$ admits a $\mathcal{C}^{k}$ parameterization with nonvanishing first derivative if and only if there are $k$ vectorvalued continuous functions

$$
B^{(N)}:[0,1] \rightarrow \mathbb{R}^{n}, \quad N=1, \ldots, k,
$$

such that

$$
\begin{aligned}
B^{(N)}\left(t_{0}\right)= & \lim _{\substack{t_{1}, \ldots, t_{2^{N-1}} \rightarrow t_{0}|| t_{p}-t_{q} \mid=o\left(\mid t_{2^{N}-1}-t_{0}\right), \forall p, q}} \frac{\sigma\left(t_{2^{N}-1}, t_{0}\right)}{\left\|\gamma\left(t_{2^{N}-1}\right)-\gamma\left(t_{0}\right)\right\|} \\
& \times \sum_{i=0}^{2^{N-1}-1} \sigma\left(t_{2 i+1}, t_{2 i}\right) \frac{\gamma\left(t_{2 i+1}\right)-\gamma\left(t_{2 i}\right)}{\left\|\gamma\left(t_{2 i+1}\right)-\gamma\left(t_{2 i}\right)\right\|} \\
& \times \prod_{s=2}^{N-1} \sigma\left(t_{2^{s}}\left(E\left[(2 i+1) /\left(2^{s}\right)\right]+1\right)-1, t_{2^{s}} E\left[(2 i+1) /\left(2^{s}\right)\right]\right) \\
& \times \frac{(-1)^{E\left[(2 i+1) /\left(2^{s}\right)\right]+1}}{\left\|\gamma\left(t_{2^{s}}\left(E\left[(2 i+1) /\left(2^{s}\right)\right]+1\right)-1\right)-\gamma\left(t_{2^{s} E\left[(2 i+1) /\left(2^{s}\right)\right]}\right)\right\|},
\end{aligned}
$$

where the precedence indicator $\sigma(\alpha, \beta)$ is 1 if $\beta<\alpha$ and -1 if $\alpha<\beta$.

PROOF. The proof makes recurrent use of arguments completely analogous to those used in the $\mathcal{C}^{2}$ case. The characterization is given by a similar but more complicated formula involving $2^{k}$ points and iterated quotients of differences of values of $\gamma$ at these points, with the corresponding precedence signs. The denominators are always of the form $\left\|\gamma\left(t^{\prime}\right)-\gamma\left(t^{\prime \prime}\right)\right\|$ for $t^{\prime}, t^{\prime \prime}$ some of these points. The formula in the statement is a compressed version, in the $\mathcal{C}^{k}$ case, of the natural formula satisfied by iterating quotients and differences of values of $\gamma$.

\section{References}

[Gar-Mar] J. Garnett and D. Marshall, Harmonic Measure, New Mathematical Monographs, 2 (Cambridge University Press, Cambridge, 2005).

[Lin] E. Lindelöf, 'Sur un principe général de l'analyse et ses applications à la théorie de la representation conforme', Acta Soc. Sci. Fenn. A 46(4) (1915), 1-35.

[Pom] Ch. Pommerenke, Boundary Behaviour of Conformal Maps, Grundlehren der Mathematischen Wissenschaften, 299 (Springer, Berlin, 1992). 
J. M. BURGUÉS, Departament de Matemàtiques, Universitat Autònoma de Barcelona, 08193 Bellaterra (Barcelona), Spain

e-mail: josep@mat.uab.cat

J. CUFÍ, Departament de Matemàtiques, Universitat Autònoma de Barcelona, 08193 Bellaterra (Barcelona), Spain

e-mail: jcufi@mat.uab.cat 